\title{
Letter
}

\section{A message in a bottle: a case report}

\author{
Felix Mehrhof ${ }^{1}$, Achim Joerres ${ }^{2}$, Rainer Dietz ${ }^{1}$ and Michael Oppert ${ }^{2}$
}

\begin{abstract}
${ }^{1}$ Charité Universitätsmedizin Berlin, Campus Virchow-Klinikum, Department of Cardiology, Augustenburger Platz, 13353 Berlin, Germany ${ }^{2}$ Charité Universitätsmedizin Berlin, Campus Virchow-Klinikum, Department of Nephrology and Medical Intensive Care Medicine, Augustenburger Platz, 13353 Berlin, Germany
\end{abstract}

Corresponding author: Felix Mehrhof, felix.mehrhof@charite.de

Published: 27 February 2008

This article is online at http://ccforum.com/content/12/1/411

(c) 2008 BioMed Central Ltd
Critical Care 2008, 12:411 (doi:10.1186/cc6784)

exhibited signs of severe peripheral polyneuropathy with initial tetraplegia, bilateral affection of the vagal nerve and resulting laryngoplegia. When he was transferred for further neurological rehabilitation after 21 days of intensive care, the patient was still suffering from occasional confusion and hallucinations and was unable to walk.

A previously healthy male physician aged 36 years presented to the emergency room because of balance disturbances and gait problems. These symptoms began a few hours after ingestion of remarkably bitter tasting mineral water in the office of the research laboratory. Soon after admission, the patient became progressively confused and reported visual hallucinations; his neurological symptoms rapidly worsened. The findings of initial diagnostic tests, including blood tests, microbiological and toxicological screening, cranial computed tomography scanning and magnetic resonance imaging, were unremarkable. Nevertheless, the patient soon had to be sedated and intubated to prevent aspiration.

Within the next 24 hours the patient developed multiple organ failure with renal, liver, respiratory and circulatory failure, and signs of disseminated intravascular coagulation and rhabdomyolysis. Severe sepsis of unknown cause was presumed, and empirical treatment with antibiotics and a course of activated protein C was initiated; clinical management required high amounts of fluids and catecholamines. Extensive microbiological tests, including repeated blood and sputum cultures, serology for leptospira and borrelia, and polymerase chain reaction for herpes simplex virus DNA, were unfruitful.

On day 3 after admission repeated toxicological examination of blood samples and the mineral water revealed contamination with acrylamide at high concentrations. Treatment with antibiotics and activated protein $\mathrm{C}$ was immediately terminated, and therapy with high doses of $N$-acetylcysteine was initiated to replenish hepatic glutathione stores. Between days 4 and 8 cardiac function gradually returned to normal, and platelet count, coagulation parameters and renal function also improved. On day 6 the patient was extubated; he
Acute acrylamide intoxication in humans is a rare event that has been reported only once in the literature [1]. The diagnosis should be considered in individuals with access to acrylamide (for instance, those working in molecular laboratories) who develop symptoms of central nervous system disturbances followed by cardiovascular, respiratory and other organ manifestations [2]. Gas chromatographic tandem mass spectrometry has been proven to be a sensitive method for the detection of acrylamide [3]. The clinical picture of acrylamide intoxication might suggest severe sepsis with acute onset, but this diagnosis should be challenged as long

\section{Competing interests}

The authors declare that they have no competing interests.

\section{Authors' contributions}

$\mathrm{FM}, \mathrm{AJ}, \mathrm{RD}$ and $\mathrm{MO}$ participated in patient treatment and preparation of the manuscript. All authors read and approved the final manuscript.

\section{Acknowledgement}

\section{References}

1. Donovan JW, Pearson T: Ingestion of acrylamide with severe encephalopathy, neurotoxicity and hepatotoxicity [abstract]. Vet Hum Toxicol 1987, 29:462.

2. International Programme on Chemical Safety Poison Information Monograph 652: Acrylamide [http://www.inchem.org/documents/pims/chemical/pim652.htm]

3. Perez HL, Osterman-Golkar S: A sensitive gas chromatography-tandem mass spectrometric method for detection of alkylating agents in water: application for acrylamide in drinking water, coffee and snuff. Analyst 2003, 128:1033-1036. as it remains a working hypothesis.

Written consent for publication was obtained from the patient. 